\title{
De la qualité de l'eau de la rivière Muko affluent du lac Edouard à travers les macroinvertébrés benthiques
}

Manuscrit reçu le 15 septembre 2015 et accepté le 10 décembre 2016

\section{Kisoholo M. A. ${ }^{1}$, Juakaly M. J-L ${ }^{2}$, Malikwisha M. D ${ }^{3}$ et MuYisa V. G ${ }^{4}$.}

${ }^{1}$ Faculté des Sciences, Université Officielle de Ruwenzori(UOR), B.P 560 Butembo, République Démocratique du Congo.

2, Université de Kisangani, B.P 2012 Kisangani, République Démocratique du Congo.

${ }^{3}$, Université Officielle de Ruwenzori, B.P 560 Butembo, République Démocratique du Congo.

${ }^{4}$ Observatoire du Système Lacustre Edouard et Albert autour du mont Ruwenzori.

\section{Résumé}

Une étude de la qualité de l'eau de la rivière MUKO, affluent du lac Edouard, à travers les macroinvertébrés benthiques a été réalisée en trois campagnes de décembre 2013 à avril 2014.

Les macroinvertébrés ont été récoltés sur les stations à l'aide de filets «SURBER» et «TROUBLEAU» respectivement pour l'analyse qualitative et l'analyse quantitative.

Les indices biologiques de cette rivière révèlent que l'eau est d'excellente qualité dans les stations 1 et 2 pour les analyses qualitative et quantitative ; à la station 3 pour l'analyse quantitative et à la station 6 pour l'analyse qualitative. Elle est de bonne qualité à la station 4 pour les analyses qualitative et quantitative; et à la station 3 pour l'analyse qualitative. Enfin, elle est de qualité modérée à la station 6 pour l'analyse quantitative.

Par ailleurs, nos résultats révèlent la présence de 490 spécimens répartis en 21 familles de macroinvertébrés reparties en 9 ordres groupés en 4 classes reparties à leur tour en 3 embranchements.

\footnotetext{
${ }^{1}$ Chef de travaux à la Faculté des Sciences, Université Officielle de Ruwenzori(UOR)

${ }^{2}$ Professeurà la Faculté des Sciences, Université de Kisangani

${ }^{3}$ Chef de travaux à la Faculté des Sciences, Université Officielle de Ruwenzori

${ }^{4}$ Chercheur à l'Observatoire du Système Lacustre Edouard et Albert
} 
Mots clés : qualité, rivière, Muko, Macroinvertébrés

\section{Summary}

A study of the water quality of MUKO River, a tributary of Lake Edward, through benthic macroinvertebrates was conducted in three seasons from December 2013 to April 2014.

Macroinvertebrates were collected on stations using «SURBER» and «TROUBLEAU» nets, respectively, for qualitative and quantitative analysis.

Biological evidence reveals that river water is of excellent quality at stations 1 and 2 for the qualitative and quantitative analysis; the station 3 for quantitative analysis and the station 6 for the qualitative analysis. It is good at station 4 for qualitative and quantitative analysis; and station 3 for qualitative analysis. Finally, it is of moderate quality at the station 6 for quantitative analysis.

Moreover, our results reveal the presence of 490 specimens of macroinvertebrates divided into 21 families divided into 9 levels grouped into 4 classes divided into three branches.

Keywords: quality, river, Muko, macroinvertebrates.

\section{Introduction}

L'espèce humaine est la première responsable de la dégradation des conditions de la viabilité de la biosphère suite d'une part à l'augmentation croissante et rapide de sa population et d'autre part à cause des progrès technologiques sans cesse croissants.

La démographie galopante ; l'usage des pesticides pour lutter contre les ennemies des cultures, des engrais chimiques pour accroître le rendement des cultures, le progrès technologique qui engendre la production des déchets non biodégradables, les produits chimiques dangereux et l'émission de nombreux gaz, etc..., sont à l'origine des différentes pollutions que connaît la planète terre[1].

Les précipitations, les mécanismes de dépôt sec des particules ramènent les polluants atmosphériques dans l'hydrosphère et/ou à la surface du sol. Le jeu du lessivage et de l'érosion hydrique, transfèrent les polluants du sol vers l'hydrosphère. Ainsi, l'hydrosphère (les océans surtout) constitue en définitive l'ultime réceptacle des agents toxiques et autres contaminants produits par la civilisation technologique [2]. 
Cependant, les polluants ramenés dans l'hydrosphère agissent défavorablement sur les écosystèmes hydrosphériques en créant une pollution pouvant avoir comme conséquences : des graves menaces à la santé des êtres vivants aquatiques, la diminution de la quantité et de la qualité de l'eau potable utilisée à l'échelle de la planète [3].

Ainsi, la structure des communautés des macroinvertébrés aquatiques est utilisée comme indicatrice des effets des activités humaines sur des écosystèmes des cours d'eau et pour fournir plus d'informations sur la qualité de l'eau et de l'habitat [4]. On peut les utiliser pour identifier plusieurs types de pollutions comme la pollution organique, métallique, de même que pour détecter une acidification du milieu [5].

Cette recherche vise d'une part à inventorier/évaluer la richesse et l'importance spécifique des macroinvertébrés tenant compte de l'altitude et d'autre part à mesurer le degré de pollution de la rivière Muko via les macroinvertébrés y inventoriés. Comme hypothèse, nous estimons que la rivière MUKO aurait une eau de bonne qualité suite à la situation de sa plus grande partie loin des activités anthropiques. Par ailleurs, les familles des macroinvertébrés aquatiques sensibles à la pollution seraient celles qui peuplent la rivière MUKO.

\section{Matériel et méthodes}

Notre recherche a été réalisée dans la rivière MUKO située à plus ou moins $60 \%$ de sa longueur dans le parc national des Virunga en République Démocratique du Congo. Il prend sa source dans les montagnes surplombant le parc, à sa partie Ouest, précisément dans la vallée de Vughusa située entre le mont Kyabirimu et le village de Burusi à 2379 m d'altitude, et à $0^{0}$ 5' 197' 'Sud, 290 26' 734' 'Est. Nous avons considéré deux sites : le site 1 à partir de la source de la rivière avec trois stations (I, II et III) et le site 2 à partir de l'embouchure de la rivière avec le lac Edouard avec aussi trois stations (IV, V et VI).

Une récolte bimensuelle s'est faite à l'aide de deux filets «SURBER» et «TROUBLEAU» ayant la maille 0,5mm. L'identification des spécimens a l'aide d'une loupe binoculaire s'est faite jusqu'à la famille en utilisant la clé de détermination décrite par Moison [6]. L'interprétation de la qualité de l'eau via les macroinvertébrés a été réalisée d'après la méthode décrite par Masson [7]. Cette méthode s'articule sur l'indice BMWP (Biological Monitoring Working Party) et ses dérivées : ASPT (Average Score Per Taxon), OQR (Overal Quality Ratting) et LQI (Lincoln Quality Indice). Elle consiste à identifier les organismes jusqu'au niveau de la famille. On se réfère au tableau standard de la détermination de l'indice BMWP (tableau 1 en annexe) qui rassemble les groupes des familles correspondant chacun à une performance (score allant de 1 à 10), le score 9 n'existe pas. Les tableaux 2 et 3 en annexe présentent les indices ASPT, OQR et LQI.

Pour mesurer la tendance centrale et la dispersion des indices biologiques calculés pour chaque station, la déviation standard (SD) a été estimée selon la formule suivante : 


$$
\mathrm{SD}=\sum \sqrt{\frac{(x i-x)^{2}}{n-1}}
$$

où SD est l'écart type [8].

Les indices utilisés impliquent différentes unités de mesures et dimensions, leur variabilité peut être comparée par leur coefficient de variabilité (CV). Un coefficient de variabilité, exprime la déviation standard ou écart type. Il est calculé selon la formule suivante :

$$
\mathrm{CV}=\frac{S D}{x} \times 100
$$

où $\mathrm{CV}$ est le coefficient de variabilité.

\section{Résultat}

\subsection{Composition faunistique}

Au cours de notre recherche, 490 spécimens de macroinvertébrés aquatiques ont été récoltés. Leur répartition selon les stations est indiquée dans le tableau 4.

Tableau 4 : Répartition des effectifs des macroinvertébrés selon les stations.

\begin{tabular}{cccccccc}
\hline Station & St I & St II & St III & St IV & St V & St VI & Total \\
\hline Effectifs & 66 & 106 & 90 & 83 & 77 & 68 & 490 \\
$\%$ & 13,46 & 21,63 & 18,36 & 16,93 & 15,71 & 13,87 & 100 \\
\hline \multicolumn{7}{c}{ Légende $:$ St = station }
\end{tabular}

Le tableau 5 présente la composition taxonomique de deux sites échantillonnés et la position systématique des familles recensées. 
Bulletin de la Société royale des Sciences de Liège, Vol. 85, 2016, p. 225 - 236

Tableau 5 : composition taxonomique de deux sites échantillonnés et position systématique des familles recensées.

\begin{tabular}{|c|c|c|c|c|c|c|c|c|c|c|c|c|c|}
\hline \multirow[t]{2}{*}{ Embranchement } & \multirow[t]{2}{*}{ Classe } & \multirow[t]{2}{*}{ Ordre } & \multirow[t]{2}{*}{ Famille } & \multicolumn{4}{|c|}{ Site I } & \multicolumn{4}{|c|}{ Site II } & \multirow[t]{2}{*}{ Tot } & \multirow{2}{*}{$\begin{array}{c}\% \\
\text { classe }\end{array}$} \\
\hline & & & & St I & St II & St III & Tot & St IV & St V & St VI & Tot & & \\
\hline \multirow[t]{17}{*}{ Arthropodes } & Insectes & Odonates & Libellulidae & - & 10 & 15 & 25 & 11 & 7 & 4 & 22 & 47 & 93,3 \\
\hline & & & Aeshnidae & 4 & 17 & 3 & 24 & 6 & 11 & 20 & 37 & 61 & \\
\hline & & & Lestidae & 52 & 57 & 34 & 143 & - & - & 2 & 2 & 145 & \\
\hline & & & Condulegasteridae & - & - & - & - & - & 1 & - & 1 & 1 & \\
\hline & & Plécoptères & Perlodidae & 2 & 1 & 6 & 9 & 3 & 2 & 6 & 11 & 20 & \\
\hline & & Heteroptères & Pleidae & - & 1 & - & 1 & 31 & 26 & 19 & 76 & 77 & \\
\hline & & & Mesovelidae & - & - & - & - & 4 & 17 & - & 21 & 21 & \\
\hline & & & Notonectidae & - & - & - & - & - & - & 1 & 1 & 1 & \\
\hline & & Coleoptères & Hydrophilidae & - & 9 & 10 & 19 & 18 & 10 & 1 & 29 & 48 & \\
\hline & & Trichoptères & Psychomydae & - & - & - & - & 1 & - & - & 1 & 1 & \\
\hline & & & Hydropsichidae & 1 & - & - & 1 & 1 & - & - & 1 & 2 & \\
\hline & & & Dipseudopsidae & - & - & - & - & 7 & 1 & - & 8 & 8 & \\
\hline & & Diptères & Ceratopogonidae & 1 & 3 & - & 4 & - & - & 3 & 3 & 7 & \\
\hline & & & Simulidae & 2 & - & 14 & 16 & - & - & 1 & 1 & 17 & \\
\hline & & Ephemeroptères & Potamanthidae & - & - & - & - & - & 1 & - & 1 & 1 & \\
\hline & Crustacés & & Astacidae & 2 & - & - & 2 & - & - & - & - & 2 & 0,6 \\
\hline & & & Carophilidae & - & - & - & - & - & - & 1 & 1 & 1 & \\
\hline \multirow[t]{2}{*}{ Mollusques } & Gastéropodes & Pilmonates & Planorbidae & 1 & - & - & 1 & - & 1 & 3 & 4 & 5 & 1,84 \\
\hline & & & Thiaridae & - & - & - & - & - & - & 4 & 4 & 4 & \\
\hline \multirow[t]{3}{*}{ Annélides } & Hirudinées & & Lumbriculidae & - & - & - & - & 1 & - & 2 & 2 & 3 & 4,28 \\
\hline & Hirudinées & & Glossiphoniidae & 1 & 8 & 8 & 17 & - & - & 1 & 1 & 18 & \\
\hline & & & Total général & 66 & 106 & 90 & 262 & 83 & 77 & 68 & 228 & 490 & 100 \\
\hline
\end{tabular}


Les résultats obtenus révèlent la présence de 490 individus récoltés sont repartis en 21 familles de macroinvertébrés, reparties en 9 ordres groupés en 4 classes reparties à leur tour en 3 embranchements. La classe des insectes est la plus importante avec 93,3\%, suivie de la classe des hirudinées avec $4,3 \%$, de celle des gastéropodes avec $1,8 \%$ et la plus rare est celle des crustacés avec $0,6 \%$.

\subsection{Indices biologiques}

Les données ci-haut de la faune récoltée ont été confrontées aux indications du tableau standard de détermination de l'indice (tableau 1 en annexe). Les indices ont été estimées nommément BMWP, ASPT, OQR et LQI. Le tableau 6 ci-dessous donne une synthèse des valeurs observées pour chacun d'eux.

Tableau 6 : Valeurs observées des indices BMWP, ASPT, OQR et LQI

\begin{tabular}{|c|c|c|c|c|c|c|c|c|c|c|c|}
\hline \multirow{3}{*}{$\begin{array}{l}\text { Indice } \\
\text { station } \\
\text { Station I }\end{array}$} & \multicolumn{5}{|c|}{ BMWP } & \multicolumn{2}{|c|}{ ASPT } & \multicolumn{3}{|c|}{ QRO } & \multirow[t]{2}{*}{ Type de filet } \\
\hline & $\mathrm{N}$ & $\mathrm{X}$ & SD & $\mathrm{CV}(\%)$ & $\mathrm{X}$ & SD & $\mathrm{CV}(\%)$ & $\mathrm{X}$ & $\mathrm{SD}$ & $\begin{array}{l}\mathrm{CV}( \\
\%)\end{array}$ & \\
\hline & 3 & 106 & 42,42 & 40,01 & 7,75 & 0,35 & 4,55 & 6 & 0,7 & 11,8 & Filet troubleau \\
\hline & & 142,5 & 152,02 & 106,62 & 7,53 & 0,748 & & 6 & 1,41 & 23,56 & Filet surber \\
\hline \multirow[t]{2}{*}{ Station II } & 3 & 187,5 & 140,71 & 75,04 & 7,75 & 0,35 & 4,56 & 6 & 1,06 & 17,66 & Filet troubleau \\
\hline & & 196 & 65,05 & 33,13 & 7,3 & 0,264 & 3,61 & 6,5 & 0,353 & 51,58 & Filet surber \\
\hline \multirow{2}{*}{$\begin{array}{l}\text { Station } \\
\text { III }\end{array}$} & 3 & 170,5 & 14,85 & 6,73 & 7,93 & 0,093 & 1,23 & 6,5 & 0.707 & 0 & Filet troubleau \\
\hline & & 63 & 9,59 & 15,22 & 7 & 0 & 0 & 4,5 & 0 & 10,87 & Filet surber \\
\hline \multirow[t]{2}{*}{ Station IV } & 3 & 48,5 & 48,01 & 98,98 & 6,74 & 1,886 & 27,98 & 5 & 3,511 & 70,02 & Filet troubleau \\
\hline & & 25,1 & 16,09 & 64,1 & 6,48 & 1,432 & 22,08 & 4,5 & 4,123 & 91,55 & Filet surber \\
\hline \multirow[t]{2}{*}{ Station V } & 3 & 42,5 & 31,51 & 74,14 & 5,75 & 1,06 & 18,5 & 4 & 1,068 & 26,7 & Filet troubleau \\
\hline & & 32 & 23.33 & 72,9 & 6,12 & $1,2 \mathrm{o} 4$ & 19,67 & 4 & 2,169 & 53,16 & Filet surber \\
\hline \multirow[t]{2}{*}{ Station VI } & 3 & 16,66 & 9,5 & 57,06 & 4,45 & 2,331 & 52,38 & 3 & 1,581 & 52,7 & Filet troubleau \\
\hline & & 57,16 & 38,14 & 66,73 & 6,45 & 1,927 & 29,64 & 5 & 1,921 & 38,42 & Filet surber \\
\hline
\end{tabular}

Légende : $\mathrm{x}$ : moyenne ; SD : écart type ; CV : Coefficient de variabilité ; $\mathrm{N}$ : nombre d'observation.

Le tableau 7 donne les valeurs « $\mathrm{OQR} »$, valeur «LQI» correspondantes et leur interprétation. 
Tableau 7 : valeur « OQR », valeur « LQI » correspondante et leur interprétation.

\begin{tabular}{ccccc}
\hline ST & $\begin{array}{c}\text { Valeur } \\
\text { OQR }\end{array}$ & $\begin{array}{c}\text { Indice LQI } \\
\text { correspondant }\end{array}$ & Interprétation & Analyse \\
\hline $\mathbf{1}$ & 6 & $\mathrm{~A}^{++}$ & Excellente qualité (non polluée) & $\mathrm{QT}$ \\
& 6 & $\mathrm{~A}^{++}$ & Excellente qualité (non polluée) & $\mathrm{QL}$ \\
$\mathbf{2}$ & 6 & $\mathrm{~A}^{++}$ & Excellente qualité (non polluée) & $\mathrm{QT}$ \\
& 6,5 & $\mathrm{~A}^{++}$ & Excellente qualité (non polluée) & $\mathrm{QL}$ \\
$\mathbf{3}$ & 6,5 & $\mathrm{~A}^{++}$ & Excellente qualité (non polluée) & $\mathrm{QT}$ \\
& 4,5 & $\mathrm{~B}$ & Bonne qualité (légèrement polluée) & $\mathrm{QL}$ \\
$\mathbf{4}$ & 5 & $\mathrm{~A}$ & Excellente qualité (non polluée) & $\mathrm{QT}$ \\
& 4,5 & $\mathrm{~B}$ & Bonne qualité (légèrement polluée) & $\mathrm{QL}$ \\
& 4 & $\mathrm{C}$ & Bonne qualité (légèrement polluée) & $\mathrm{QT}$ \\
& 4 & $\mathrm{C}$ & Bonne qualité (légèrement polluée) & $\mathrm{QL}$ \\
& 3 & $\mathrm{E}$ & Qualité modérée (légèrement polluée) & $\mathrm{QT}$ \\
& 5 & $\mathrm{~A}$ & Excellente qualité (non polluée) & $\mathrm{QL}$ \\
\hline
\end{tabular}

Légende : QT : Analyse quantitative ; QL : Analyse qualitative ; LQI : Lincoln Quality Indice ; OQR : Overal Quality Ratting

\section{Discussion}

\subsection{Composition faunistique}

Nos résultats révèlent la présence de 490 spécimens répartis en 21 familles de macroinvertébrés réparties dans trois embranchements (Arthropodes, Mollusques et, Annélides). Les familles des Lumbriculidae, Planorbidae et Thiaridae sont des familles indicatrices de la pollution et ont été récoltées en faible quantité. Une étude similaire a été réalisée dans le bassin d'Ishungu au Sud Kivu en République Démocratique du Congo [9]. 16 familles ont été identifiées et reparties dans trois embranchements obtenus aussi dans nos résultats (Arthropodes, Annélides et Mollusques). Une autre étude a été réalisée dans la baie de Nyakalengwa à Idjwi au Sud Kivu [10]. Cette étude a révélé qu'au totale 418 spécimens de macroinvertébrés ont été récoltés dans deux sites. Ces spécimens sont repartis dans deux embranchements (Arthropodes et Mollusques), trois classes (Insectes, Crustacés et Gastéropodes), Cinq ordres et 26 familles. Par ailleurs, une autre étude a été réalisée au niveau de dix rivières en ville de Butembo au Nord Kivu en Republique Democratique du Congo [11]. 3898 spécimens ont été récoltés et répartis dans 11 familles, 10 ordres, 3 classes et 3 embranchements. KANKONDA [12], dans sa contribution à l'établissement d'une carte de pollution des eaux de ruisseaux de Kisangani par l'utilisation des macroinvertébrés benthiques comme bioindicateurs, sur 9 familles qui sont; Hydrophylidae, Ditiscidae, Libellulidae, Gomphidae, Boetidae, Corixidae, Chironomidae et Planorbidae; seul 2 familles soit $9 \%$ de nos familles y figurent. Il s'agit de la famille des Hydrophylidae et celle de Libellulidae. Les familles des Hydropsychidae et des Thiaridae figurent parmi celles 
recensées en Guadeloupe [13]. Par ailleurs, en plus de ces deux familles, les Libellulidae, les Mesoveliidae, les Hydrophilidae ont été recensées dans des rivières de la Guadeloupe [14].

Dans tous les cas, les Arthropodes en général et particulièrement les insectes restent dominants. En effet, les insectes aquatiques forment le groupe le plus diversifié des macroinvertébrés benthiques dulcicoles et représentent $70 \%$ des espèces connues des grands groupes de macroinvertébrés aquatiques [15] et [16].

Nous référant au tableau 5 , les familles très sensibles à la pollution sont plus représentées au site I qu'au site II. Ce sont les Aeshnidae, les Libellulidae, les Lestidae.

\subsection{Indices biologiques}

\subsubsection{L'indice BMWP (Biological Monitoring Working Party)}

La rivière Muko a affiché l'indice BMWP variant entre 16,6 et 187,5 pour l'analyse quantitative et entre 32 et 196 pour l'analyse qualitative. Les valeurs variant entre 11 et 23,3 pour l'analyse quantitative et entre 7,3 et 14,4 pour l'analyse qualitative ont été enregistrées pour les rivières Kanyangoko et Makamba à Butembo. La rivière Muko affiche des valeurs supérieures par rapport aux rivières de Butembo. Cela s'expliquerait par des activités anthropiques moins intenses au niveau de cette rivière.

\subsubsection{L'indice ASPT}

Cet indice dérivant du précédent a affiché pour la rivière Muko des valeurs oscillant entre 4,45 et 7,93 pour l'analyse quantitative et entre 6,12 et 7,53 pour l'analyse qualitative.

\subsubsection{Indice $O Q R$}

Nous référant au tableau 7, les valeurs OQR de nos stations s'étendent entre 3 et 6,5 pour l'analyse quantitative et entre 4 et 6,5 pour l'analyse qualitative. Pour MUHESI en ville de Butembo [17], ces valeurs sont entre 2,5 et 4,5 pour l'analyse quantitative et entre 2 et 3,6 pour l'analyse qualitative. Par contre pour PALUKU en ville de Butembo [11], ces valeurs sont entre 1 et 5 . Nos valeurs restent généralement élevées, probablement parce que le cours d'eau reste peu impacté par la population. Le fait que nos valeurs restent élevées serait due à la position de la rivière investiguée en plein parc, surplombée par des collines à l'absence presque d'activités anthropiques.

\subsubsection{Indice LQI}

En nous basant sur le tableau3 en annexe, où LQI égal à $\mathrm{A}$ ou $\mathrm{A}^{+}$ou $\mathrm{A}^{++}$traduit une eau d'excellente qualité, un LQI égal à $\mathrm{B}$ ou $\mathrm{C}$ traduit une bonne qualité, un LQI égal à $\mathrm{D}$ ou $\mathrm{E}$ traduit une qualité modérée, un égal à $\mathrm{F}$ ou $\mathrm{G}$ traduit une qualité pauvre et celui de $\mathrm{I}$ ou $\mathrm{H}$ une très pauvre qualité. Considérant l'analyse quantitative, quatre stations sont d'excellente 
qualité (station I, II, III et IV) ; la station V est de bonne qualité et la station VI est de qualité modérée. Par ailleurs, pour l'analyse qualitative, trois stations sont d'excellente qualité, il s'agit de la station I, II et VI et trois stations sont de bonne qualité (station III, IV et V) ; notre première hypothèse est donc vérifiée. Pour MUHESI [17], la qualité de l'eau des rivières investiguées va de modérée à pauvre. Pour PALUKU [11], il se montre que la valeur OQR de toutes les stations s'étend de A à I. Ce qui se traduit, en général, par une eau de mauvaise qualité à cause des activités anthropiques intenses non loin de ces rivières.

La qualité de l'eau est la résultante des activités dont elle fait l'objet. Une eau moins sollicitée par les activités anthropiques garde une bonne qualité, ce qui se traduit par une grande diversité spécifique des macroinvertébrés tandis qu'une eau très sollicitée par l'activité anthropique perd sa qualité ainsi que sa diversité spécifique en macroinvertébrés [18].

\section{Conclusion}

L'objectif général de cette recherche était de mesurer le degré de pollution de la rivière Muko via les macroinvertébrés benthiques.

Pour atteindre nos objectifs, les macroinvertébrés ont été récoltés à l'aide de filet troubleau et de filet surber respectivement pour l'analyse qualitative et l'analyse quantitative. La recherche s'est réalisée bimensuellement de décembre 2013 jusqu'en avril 2014.

Les résultats obtenus révèlent la présence de 490 individus récoltés, en 21 familles de macroinvertébrés reparties en 9 ordres groupés en 4 classes reparties à leur tour en 3 embranchements. La classe des insectes est la plus importante avec 93,3\%, suivie de la classe des hirudinées avec $4,3 \%$, de celle des gastéropodes avec $1,8 \%$ et la plus rare est celle des crustacés avec $0,6 \%$.

Les familles des Lumbriculidae, Planorbidae et Thiaridae sont des familles indicatrices de la pollution et ont été récoltées en faible quantité. La qualité de l'eau de la rivière investiguée est encore bonne nous référant à l'indice LQI.

Eu égard aux résultats observés, nous recommandons aux futurs chercheurs que cette étude soit élargie au niveau de toutes les rivières de cette région et cela d'une manière régulière, au moins pour une année, pour que la carte de pollution y afférente soit établie, surtout que c'est une zone faisant partie du Parc National des Virunga.

\section{Références bibliographiques}

[1]. Duvignaud, P., 1980. La synthèse écologique. Populations, Communautés, Écosystèmes, Biosphère, Noosphère. Doin, 2 édition, Paris, 380 p.

[2]. Ramade F., 2010. Introduction à l'Écotoxicologie, fondement et applications, Éd TEC/ ET DOC/LAVOISIER, 618p

[3]. http://wwz.ifremer.fr/ , consulté le 26 janvier 2014.

[4]. Woodcock T. and Huryn, 2007. The response of macroinvertebrates production to a pollution gradient in hears water stream fresh biology. 
[5]. Camargo, J. A., Alonso, A. et De La Puente, M. 2004. Multimetric assessment of nutrient enrichment in impounded rivers based on benthic macroinvertebrates.Environmental Monitoring and Publishers, 96:233-249.

[6]. Moison J., 2010. Guide d'identification des principaux macroinvertébrés benthiques d'eau douce du Québec, Éd. Ministère du développement durable de l'environnement et des parcs, $338 \mathrm{p}$.

[7]. Masson, 1991. Biology of fresh water pollution, ed. Longman scientific and technical, $338 \mathrm{p}$.

[8]. Lind T, 1985. Hand book of common method in limnology and kendoll hunt, 199 p.

[9]. Aksanti, B., 2010. Contribution à la structure des communautés des macroinvertébrés benthiques du lac Kivu, Bassin d'Ishungu, mémoire, inédit ISP/Bukavu, 30 p.

[10]. Sakwe B. D., 2011. Contribution à l'étude d'impact des activités anthropiques sur les peuplements macrobenthiques littoraux du lac Kivu : cas de la baie de Nyakalengwa à Idjwi., TFC inédid, ISP IDWI, $26 \mathrm{p}$.

[11]. Paluku M. I., 2011. Contribution à l'évaluation des indices biologiques basés sur les macroinvertébrés aquatiques dans la détermination de la qualité de l'eau, cas de 10 rivières de la ville de Butembo mémoire inedit, UOR, 44 p.

[12]. Kakonda B. A., 2001 : Contribution à l'établissement d'une carte de pollution des eaux des ruisseaux de Kisangani par l'utilisation des macroinvertébrés comme bioindicateurs, Dissertation inédite. Fac SC UNIKIS, 54 p.

[13]. Touron P., Bernadet C., Compin A., Bargier N., and Céréghino R., 2013. River classification as the bassin for freshwater biological assessment in overseas Europe: issues raised from Guadeloupe (French Lesser Antilles). International Review of Hydrobiology, 98, 34-43.

[14]. Touron P., 2014 : Biodiversité des communautés d'invertébrés benthiques des rivières de la Guadeloupe et réponses aux perturbations anthropiques, Thèse de Doctorat, Université de Toulouse, $182 \mathrm{p}$.

[15]. Afnor, 2009. Qualité de l'eau - Traitement au laboratoire d'échantillons contenant des macroinvertébrés de cours d'eau. XP T90-388, Association Française de Normalisation, Paris, $21 \mathrm{p}$.

[16]. Hofmann C., Sartori M. et Thomas A., 1999. Les Éphéméroptères de la Guadeloupe (Petites Antilles Françaises). Mémoire de la Société Vaudoise des Sciences Naturelles, 20 :196.

[17]. Muhesi K., 2010. Contribution à l'évaluation des indices biologiques basés sur les macroinvertébrés aquatiques dans la détermination de la qualité de l'eau, cas des rivières Kanyangoko et Makamba en ville de Butembo, mémoire inédit, UOR, 41 p.

[18]. Aude B., 2008 : Bio-évaluation de la qualité de l'eau : établissement d'un protocole d'échantillonnage simplifié, basé sur la collecte des macroinvertébrés benthiques sur les seuils des rivières à charge de fond graveleuse. Biodiversity and Ecology. Université Blaise Pascal Clermont Ferrand II. 
Bulletin de la Société royale des Sciences de Liège, Vol. 85, 2016, p. 225 - 236

\section{Annexes}

Tableau 1 : Tableau standard de détermination de l'indice BMWP

\begin{tabular}{|c|c|c|}
\hline ORDRE/CLASSE & FAMILLES & SCORE \\
\hline EPHEMEROPTERES & Siphlonuridae, heptageniidae, ephemerellidae, ephemeridae, leptophlebeidae. & \\
\hline PLECOPTERES & Taeniopterygidae, leuctridae, capnidae, perlodidae, perlidae, chloroperlidae. & \\
\hline & & \\
\hline HETEROPTERES & Aplelocheiridae & \\
\hline TRICOPTERES & $\begin{array}{l}\text { Phryganeidae, Molannidae, Odontoceridae, Lepidomatidae, Goeridae, } \\
\text { Sericostomatidae, Leptoceridae. }\end{array}$ & \\
\hline CRUSTACES & Astacidae. & 8 \\
\hline ODONATES & $\begin{array}{l}\text { Lestidae, Agriidae,Gomphidae,Cordulegasteridae, Aeschnidae , cordilliidae, } \\
\text { Libellulidae }\end{array}$ & \\
\hline TRICOPTERES & Psychomyidae, Phylopotamidae & \\
\hline EPHEMEROPTERE & Caenidae & 7 \\
\hline PLECOPTERE & Normovidae & \\
\hline TRICHOPTERE & Rhyacophylidae, polycentropidae, limnephilidae & \\
\hline GASTEROPODE & Neritidae, Viviparidae, Ancylidae, & 6 \\
\hline TRICHOPTERE & Hydroptilidae & \\
\hline PELECYPODES & Renionidae & \\
\hline CRUSTACES & Corophiidae, Gammaridae & \\
\hline ODONATES & Platycnemidae, Coenagriidae & 5 \\
\hline HETEROPTERES & $\begin{array}{l}\text { Mesoveliidae, Hygrobiidae, Gerridae, Nepidae, Naucoridae, Notonectidae, } \\
\text { Pleidae, Corixidae. }\end{array}$ & \\
\hline COLEOPTERES & $\begin{array}{l}\text { Haliphidae, Hydrobiidae, Dytixidae, Gyrinidae, Hydrophilidae. Clambidae, } \\
\text { Helodidae, Dryopidae, Chrysomelidae, Curculionidae }\end{array}$ & \\
\hline TRICOPTERES & Hydropsychidae & \\
\hline DIPTERES & Tipilidae, Simuliidae & \\
\hline PLATHELMINTHES & Planariidae, Dendrocoelidae & \\
\hline EPHEMEROPTERES & Baetidae, potamathidae & 4 \\
\hline MEGALOPTERES & Sialidae & \\
\hline HIRUDINES & Pixicolidae & \\
\hline GASTEROPODES & Valvatidae,hydrobidae,Limnaeidae,Plysidae, Planorbidae, Thiaridae & 3 \\
\hline PELECYPODES & Sphaeriidae & \\
\hline HIRUDINES & Glossiphonidae, Erpobdellidae & \\
\hline CRUSTACES & Asellidae & \\
\hline DIPTERES & Chironomidae & 2 \\
\hline OLIGOCHETES & Toute la classe & 1 \\
\hline
\end{tabular}

Source : [7] 
Bulletin de la Société royale des Sciences de Liège, Vol. 85, 2016, p. 225 - 236

Tableau 2 : Valeurs standard dérivées des scores BMWP et ASPTS

\begin{tabular}{cccc}
\hline BMWP & Valeur X & ASPT & Valeur Y \\
\hline $\mathbf{1 5 1}$ & 7 & $6,0+$ & 7 \\
$\mathbf{1 2 1}-\mathbf{1 5 0}$ & 6 & $5,5-, 9$ & 6 \\
$\mathbf{9 1 - 1 2 0}$ & 5 & $5,1-5,4$ & 5 \\
$\mathbf{6 1 - 9 0}$ & 4 & $4,6-5,0$ & 4 \\
$\mathbf{3 1 - 6 0}$ & 3 & $3,6-4,5$ & 3 \\
$\mathbf{1 5}-\mathbf{3 0}$ & 2 & $2,6-3,5$ & 2 \\
$\mathbf{0 - 1 4}$ & 1 & $0-2,5$ & 1 \\
\hline
\end{tabular}

Source : [7]

Tableau 3 : Valeurs OQR, LQI correspondantes et leurs interprétations

\begin{tabular}{ccc}
\hline Valeur OQR & Indice LQI & Interprétation \\
\hline $\mathbf{6}$ & $\mathrm{A}++$ & \\
$\mathbf{5 , 5}$ & $\mathrm{A}+$ & Excellente qualité \\
$\mathbf{5}$ & $\mathrm{A}$ & Bonne qualité \\
$\mathbf{4 , 5}$ & $\mathrm{B}$ & \\
$\mathbf{4 , 0}$ & $\mathrm{C}$ & Qualité modérée \\
$\mathbf{3 , 5}$ & $\mathrm{D}$ & \\
$\mathbf{3 , 0}$ & $\mathrm{E}$ & \\
& & Qualité pauvre \\
$\mathbf{2 , 5}$ & & Très pauvre qualité \\
$\mathbf{2 , 0}$ & $\mathrm{F}$ & \\
$\mathbf{1 , 5}$ & $\mathrm{G}$ & \\
$\mathbf{1 , 0}$ & $\mathrm{H}$ & $\mathrm{I}$ \\
& Source : [7] & \\
& &
\end{tabular}

